\title{
Adult Health Nursing
}

National Cancer Institute

\section{Source}

National Cancer Institute. Adult Health Nursing. NCI Thesaurus. Code C18617.

A subdiscipline of nursing focusing on general and comprehensive nursing care directed to adults. 